\title{
DIFERENCIAS ENTRE WHEWELL Y HERSCHEL RESPECTO A LA IDEA DE CAUSALIDAD HISTÓRICA: UN CASO SOBRE EL INTRINCADO DESARROLLO DE LA METODOLOGÍA*
}

GODFREY GUILLAUMIN

FACULTAD DE FILOSOFÍA Y LETRAS

UNIVERSIDAD DE GUANAJUATO

\section{Introducción}

Una gran parte de la literatura contemporánea sobre filosofía de la ciencia gira alrededor del problema de la explicación científica. Se han establecido diversas estrategias con el fin de determinar cuáles son las condiciones necesarias de las explicaciones científicas y cuándo tenemos buenas razones para aceptarlas. Algunos de los principales intentos en esta dirección son los trabajos de Hempel Salmon y van Fraassen. En este artículo me propongo arrojar luz sobre un aspecto del problema de la explicación que no ha recibido suficiente atención, a saber, la manera en que diversos supuestos filosóficos intervienen en la evaluación de explicaciones científicas de teorías nuevas. Pretendo analizar en detalle un episodio de la historia de la ciencia para apoyar la tesis de que, en gran medida, parte de los criterios para evaluar una explicación científica son extraídos de la manera de concebir el desarrollo histórico de metodologías exitosas.

El episodio que examinaré es el surgimiento de la geología de Lyell hacia la primera mitad del siglo xx. Especificamente me detendré en analizar las evaluaciones que realizaron $\mathrm{J}$. Herschel y W. Whewell sobre el tipo de explicaciones que Lyell proveía en el marco de la metodología de las causas verdaderas. Mientras que para Herschel la teoría de Lyell, particularmente la teoría del clima, presentaba todas las credenciales para ser considerada una buena teoría, para Whewell habría que tomar en cuenta otras consideraciones metodológicas antes de depositar una confianza similar en tal teoría.

Sostendré la tesis de que las diferencias entre Whewell y Herschel se encuentran no sólo en cómo entienden una causa verdadera, sino más bien

* Deseo agradecer a Larry Laudan, Rachel Laudan y a Sergio Martínez las útiles y estimulantes y discusiones y críticas hechas durante mi trabajo sobre este artículo. 
en que aluden a un conjunto de problemas que Whewell veía en las explicaciones de las ciencias que integran causalidad histórica. Me refiero a problemas epistemológicos respecto a cómo entender y validar la causalidad histórica en las teorías de las ciencias "históricas" y la forma en que otros elementos, como la clasificación, la no predicción, la noción de complejidad, etc., se relacionan con la causalidad histórica en el pensamiento de Whewell. Este episodio muestra claramente que determinar cuándo algo es una explicación científica genuina depende de múltiples consideraciones filosóficas alrededor de diversos temas.

La estrategia que voy a seguir es exponer de manera breve algunos de los problemas filosóficos y empíricos con los cuales Lyell se enfrentó al elaborar su teoría geológica. Después analizaré las críticas metodológicas de Herschel a la teoría de Lyell a la luz de la relación que Herschel establece entre leyes naturales y causalidad. Pasaré después a examinar la noción whewelliana de ciencias palætiológicas con el fin de enfatizar su relevancia filosófica y epistemológica. Finalmente, concluiré acentuando algunas diferencias fundamentales entre Herschel y Whewell acerca de estos temas. Cabe mencionar que este tópico de la historia y filosofía de la metodología no ha sido suficientemente explorado y parte de mi propósito aquí es cubrir este vacío.

2. La geología de Lyell: rechazo a las hipótesis y articulación de causalidad histórica

A finales del siglo xvir y durante las primeras décadas del xix el problema de explicar fenómenos que involucraban historicidad se presentó con cierta fuerza en diferentes disciplinas. La lingüística alemana, la geología, la embriología, la historia humana, etc., involucraban de manera distinta la dificultad de describir diferentes procesos mediante los cuales los fenómenos que les competían habían llegado al estado actual. A. de Candolle (1778-1841), J. Hutton (1726-1797), J. Blumenbach (1752-1840), J.B. de Lamarck (1744-1829), A.G. Werner (1749-1817), G. Cuvier (1769-1832), Ch. Lyell (1797-1875), W. Whewell (1794-1866), etc., son algunos de los hombres de ciencia involucrados en problemas que introducían la historicidad de diversas formas.

La teoría geológica de Lyell, publicada en 1830-1833 con el título de Principles of Geology, aparece como un caso particular de este ambiente problemático que tiene como marco la explicación histórica. Uno de los principales problemas involucrados en este tipo de explicación consistía en dificultades teóricas de la clasificación en diferentes áreas como la botánica, la mineralogía, la lingüística, etc. Específicamente dichas dificultades residían, en gran parte, en el tipo de relaciones o afinidades que se debian 
buscar o adoptar en los diversos sistemas naturales. Incluso en cómo se entendía "sistema natural". 1

Una de las metas de Lyell fue elaborar una teoría geológica que no fuera especulativa como las dominantes en su tiempo. Las teorías geológicas de Werner y Cuvier apelaban a fenómenos desconocidos - grandes catástrofes o la acción de un centro terrestre de calor - y ello Lyell lo consideraba hipotético. El problema epistemológico principal que Lyell veía en las teorías rivales era cómo saber si esos fenómenos postulados realmente existieron. Postulando esos fenómenos era posible explicar diversos fenómenos geológicos como cierto ordenamiento de la estratigrafía, fósiles encontrados en altas montañas, formación rocosa, etc. La estrategia de Lyell fue articular algunos de los principios geológicos de Hutton (Hutton 1788) en términos de causas verdaderas (verae causae).

El principio de vera causa fue una herencia metodológica de Newton basado fundamentalmente en su primera regla para filosofar; la cual dice que "no deben admitirse más causas de las cosas naturales que aquellas que sean verdaderas y suficientes para explicar sus fenómenos". Lyell articuló ese principio asumiendo un desarrollo uniforme de los sucesos geológicos. Él pensó que "las leyes de la naturaleza no han cambiado a través del tiempo" (Laudan 1987, p. 205) y que sólo los fenómenos como los terremotos, acción volcánica, sedimentos, movimientos de los océanos, etc., son las únicas causas geológicas aceptadas en su geología no hipotética. Al principio de su tercer volumen de los Principles, Lyell dice que "en nuestro intento por desenmarañar esas cuestiones [geológicas], deberemos adoptar un camino diferente, restringiéndonos a las operaciones conocidas o posibles de causas existentes" (Lyell 1830-1833, vol. III, p. 6). De igual forma, mantuvo que tales causas o fuerzas actualmente operando son del mismo tipo que aquellas que han estado operando en el pasado y hasta el presente, y que su magnitud ha sido la misma en todos los tiempos. A esas dos características básicas de su teoría, Whewell las llamó actualismo y uniformismo respectivamente. ${ }^{2}$ Lyell expresó la idea del actualismo en el subtítulo de sus Principles como "un intento de explicar los cambios primigenios de la superficie de la Tierra mediante la referencia a causas ahora en operación".

1 Una distinción conceptual importante en esta época fue la desarrollada por I. Kant (1724-1804) quien distinguió las clasificaciones que se basaban en relaciones contemporáneas de similitud y aquellas que se basaban en un concepto histórico y genealógico de relación orgánica. De tal forma que el aspecto genealógico o histórico estaba amalgamado con el problema de la clasificación. La teoría de Lyell incorporará este tipo de problemas.

2 La noción de uniformismo es una noción mucho más compleja que la aquí expuesta, algunos estudiosos han encontrado básicamente tres sentidos (cfr. Rachel Laudan 1987, p. 205). No obstante, para mis fines me basta con el alejamiento de las hipótesis de la posición de Lyell. 
La estrategia metodológica de Lyell fue exitosa para explicar el gradual cambio global del clima. Él mostró cómo las diferentes proporciones de distribución entre el mar y la tierra firme hacen que el clima global varíe. Esta teoría asumía que las diferencias en dicha distribución se debían a que los continentes se elevaban muy por encima del nivel del mar, hecho respaldado por las conchas marinas fosilizadas encontradas en altas montañas. Sin embargo, enfrentó algunas dificultades para explicar la elevación de los continentes mediante una teoría de vera causa. Una de tales dificultades fue que Lyell no pudo encontrar ninguna causa verdadera (actualmente observada) responsable de la elevación de los continentes. Enfrentado con ese problema él podría haber elaborado hipótesis mediante causas desconocidas, si no hubiera seguido la metodología de la vera causa para explicar tal fenómeno; no obstante no recurrió a hipótesis porque "el método por hipótesis, pensaba Lyell, no era confiable; en consecuencia evitó hipótesis acerca de las causas de los volcanes y terremotos tanto como pudo" (Laudan 1987, p. 217). Rechazó el método hipotético arguyendo que el método correcto de análisis consiste en inferir el pasado del presente. La tesis básica aquí es que "todas las hipótesis acerca de causas o efectos inobservables deben estar firmemente fundadas en aquello que hemos observado" (Laudan 1987, p. 204). En un párrafo de sus Principles, Lyell clarifica este punto:

En lugar de conjeturas vagas, como aquello que ha sido establecido del planeta en la época de su creación, nosotros centramos nuestros pensamientos firmemente en la conexión presente entre el clima y la distribución de la tierra firme y el mar [...] podemos así aproximarnos a una teoría verdadera. Si alguna duda aún persiste, ésta debe ser adscrita a nuestra ignorancia de las leyes de la naturaleza. (Lyell 1830, vol. I, p. 105)

Es crucial notar que el método de vera causa, de acuerdo con Lyell, era un medio para obtener conocimiento verdadero sobre problemas geológicos sólo bajo el supuesto de que la historia pasada de la Tierra nunca ha sido fundamentalmente diferente de aquello que podemos observar en el presente. El presente no sólo es la llave del pasado sino también la clave del futuro, porque las leyes de la geología han sido, y serán, siempre del mismo tipo; ellas gobiernan y continuarán gobernando los fenómenos geológicos de la misma forma que en el pasado. En realidad Lyell no se interesó por el futuro, pero es igualmente claro que su principio uniformista se puede extender hacia el futuro principalmente porque las leyes de la naturaleza son invariables y universales. De acuerdo con Lyell, el cambio geológico es constante, lo cual hace que la Tierra se encuentre en balance, i.e., en estado de equilibrio estable. Gould (1965, 1987) y Rudwick (1972) han señalado que Lyell utilizó su principio uniformista en cuatro sentidos diferentes. 
Aquí no voy a criticar sus puntos de vista, sino que sólo mencionaré uno de tales sentidos con el fin de arrojar alguna luz sobre el punto que me interesa.

El sentido de uniformidad en el cual estoy interesado sostiene que "las leyes naturales son constantes en espacio y en tiempo. Los filósofos han reconocido desde siempre [...] que los supuestos acerca de la invariabilidad de las leyes naturales sirven como una garantía necesaria para extender las inferencias inductivas en un pasado inobservado" (Gould 1987, p. 119). De acuerdo con Lyell, los fenómenos geológicos son gobernados por leyes de la naturaleza; sostiene que "la geología es la ciencia que investiga los cambios sucesivos que han acontecido en el reino orgánico e inorgánico de la naturaleza" (Lyell 1830, vol. I, p. 1). A pesar de la relación de la geología con las leyes invariables de la naturaleza, algunos de los rasgos epistemológicos de tales leyes no se presentan en la geología, por ejemplo, la predicción en el sentido de la mecánica celeste. Lyell era perfectamente consciente de las diferencias entre la geología y las ciencias como la mecánica celeste; particularmente consideraba el carácter distintivo de la investigación histórica. Para él la explicación de fenómenos presentes integra resultados contingentes de un pasado que pudo haber sido diferente, en este sentido no articula resultados predecibles mediante leyes de la naturaleza (Gould 1987). Lyell traza la mencionada diferencia en los siguientes términos:

Los geómetras han medido las regiones del espacio y las distancias relativas de los cuerpos pesados. Los geólogos han estimado miriadas de eras no mediante el cálculo aritmético, sino por un tren de sucesos físicos, una secuencia de fenómenos en el mundo animado e inanimado, signos que transmiten a nuestras mentes ideas, más definidas de lo que nos podemos figurar, acerca de la inmensidad del tiempo. (Gould 1987, p. 154)

Lyell enfatiza que la historia debe ser una sucesión de fenómenos, donde cada uno de ellos obedece las leyes de la naturaleza, y la secuencia contingente de todos constituye el carácter histórico de la geología. Así, para Lyell, la geología tiene un aspecto empírico en la medida en que trabaja con causas verdaderas y leyes de la naturaleza. Herschel evaluó favorablemente la teoría de Lyell porque seguía principios de vera causa, pero principalmente porque se ajustaba a cierta idea herscheliana de la relación entre causas y leyes.

\section{John FW. Herschel y la aceptación de la teoría de Lyell}

En un célebre artículo que escribió hacia finales de la década de 1820 para la Encyclopedia Metropolitan titulado "Luz", J. Herschel (1792-1871) ya 
había desarrollado en líneas generales lo que más tarde articularía en el Preliminary Discourse como vera causa. Uno de los estudiosos de Herschel, Good, sostiene que "es aquí [en el artículo 'Luz'] que Herschel explícitamente utiliza el término vera causa por primera vez. Lo utiliza en su discusión de la explicación ondulatoria de Fresnel sobre los colores producidos por la luz polarizada en cristales" (Good 1987, p. 7). Herschel ${ }^{3}$ codificó sus procedimientos y estándares metodológicos utilizados en sus trabajos de óptica de 1819 y 1820 en su Preliminary Discourse de 1830, particularmente en la sección "Primera etapa inductiva". Esta relación entre sus trabajos en óptica y algunas de sus ideas metodológicas será un factor importante, aunque no exclusivo, para vincular su idea de las leyes de la naturaleza con el papel metodológico que desempeñaban en las explicaciones. Como veremos, para Herschel no hay una distinción fundamental respecto al papel epistemológico de las leyes en óptica y en geología.

Respecto al origen y a diferentes características de las causas verdaderas, Herschel sostiene que:

la experiencia nos ha mostrado la manera en que un fenómeno depende de otro en una gran variedad de casos. Así nos encontramos provistos, en la medida en que la ciencia se extiende, con una cantidad en continuo aumento de tales fenómenos antecedentes o causas competentes, bajo diferentes modificaciones, para la producción de una gran multitud de efectos, además de aquellos que originalmente guiaron al conocimiento de ellas. A tales causas Newton les aplicó el término de verae causae, esto es, causas reconocidas que tenían una existencia real en la naturaleza y no eran meras hipótesis o inventos de la mente. (Herschel 1987, pp. 144-145)

Si bien en esta primera aproximación a las causas verdaderas son dos las condiciones que reconoce para que una causa sea verdadera -que realmente exista y que no sea un invento de la mente-la principal motivación metodológica de Herschel es evitar hipótesis en filosofía natural. ${ }^{4}$ La evaluación favorable de Lyell por parte de Herschel viene dada principalmente porque contaba con apoyo empírico positivo, mientras que las teorías geológicas rivales utilizaban especulaciones; además de que las causas aducidas por él eran adecuadas y suficientes. "Apoyo empírico positivo" puede ser

3 La más importante y original investigación de Herschel en óptica fue realizada entre 1819 y 1820 . Estuvo dedicada a descubrir los axiomas o leyes físicas de las cuales uno puede deducir los patrones de colores particulares producidos cuando la luz polarizada es trasmitida a través de los cristales con uno o dos ejes ópticos (cfr. Buchwald 1989, p. 291).

4 Algunos estudiosos de Herschel se han dado a la clarificación de las condiciones que él imponía a la primera condición para que una causa fuera verdadera -realmente existentey han enfatizado que esa condición es complementada con las condiciones de suficiencia y adecuación. 
entendido como la mínima condición necesaria para considerar una causa como vera causa; éste consiste en apoyar la teoría propuesta con evidencia empírica que proviene de hechos conocidos. Veamos esto con más detalle.

Refiriéndose a la teoría del clima de Lyell, Herschel sostiene que:

Tenemos aquí, por tanto, una causa evidente y real, de suficiente universalidad y actuando en la dirección correcta para explicar el fenómeno. Su adecuación es otro punto. (Herschel 1987, p. 148)

Herschel evaluó favorablemente la teoria de Lyell principalmente porque "ninguna de ellas [teorías rivales] pueden ser consideradas como causas reales en el sentido aquí defendido, porque no sabemos que el Globo se ha enfriado por fusión, ni estamos seguros de que una gran actividad tanto de los primeros volcanes como de los presentes haya realmente existido" (Herschel 1987, p. 146). Contrariamente, "la elevación del fondo del mar para llegar a ser tierra firme ha sido testificado realmente de manera frecuente y a tal escala como para calificarla como una vera causa disponible en la filosofía sólida" (Herschel 1987, p. 144). Refiriéndose a las opciones explicativas, afirma que en la teoría de Lyell "tenemos una causa real y evidente de suficiente universalidad y actuando en la dirección correcta para explicar el fenómeno" (Herschel 1987, p. 147).

De la naturaleza epistémica de las diferentes teorías geológicas anteriores a Lyell, Herschel sostiene que "ellas, en verdad, fueron las especulaciones favoritas de una raza de geólogos ahora en extinción, pero la ciencia en sí misma ha sufrido un cambio total de carácter, incluso dentro de la última mitad del siglo pasado y ha sido traída, en gran medida, eficazmente dentro de la lista de las ciencias inductivas" (Herschel 1987, p. 282; las cursivas son mías). Pero existe otra razón en Herschel para colocar la geología en la lista de las ciencias inductivas, aparte del hecho de que ésta no recurre a hipótesis. Ésta otra razón se refiere a la manera en que las causas operan. De acuerdo con Herschel, las explicaciones legítimas deben ser causales y en él hay un sentido claro sobre qué significa que las explicaciones sean causales. ${ }^{5}$ Sostiene que "la primera cosa que una mente filosófica considera cuando un nuevo fenómeno se presenta, es su explicación o referencia a una causa inmediata" (Herschel, 1987, p. 144). Aquí Herschel distingue entre lo que llama causas próximas y causas últimas, pero lo que me interesa enfatizar es que una relación causal, sea próxima o última, está caracterizada por las siguientes reglas: "Primera, conexión invariable...; Segunda,

5 Ya Biacke ha determinado los tres sentidos en que Herschel entiende lo que es una causa. En ese trabajo se enfatiza el tercer sentido como el principal que asume Herschel para su teoría de la ciencia, aquí seguiré ese tercer sentido (Blacke 1960). 
negación invariable...; Tercera, incremento o disminución del efecto...; Cuarta, proporcionalidad y, Quinta, reversión del efecto" (Herschel 1987, pp. 151-152). La causalidad expresada en esas reglas tiene como eje la idea de invariabilidad y de nexo necesario, lo cual supone un sustrato ahistórico de la acción de la causa. El transcurso del tiempo no altera el efecto que ha de esperarse cuando se presenta una causa específica. En otras palabras, la acción de la causa es independiente del momento en que se aplique. En caso de presentarse ciertas causas, y sin nada que impida su natural acción, un efecto determinado será esperado. A ese tipo de causalidad Whewell la llamará, como veremos, "causalidad permanente".

El carácter ahistórico o atemporal de la concepción de causalidad de Herschel está en la base de la relación que establece entre causas y leyes de la naturaleza. Si bien esta relación es oscura, tiene gran importancia para el punto que interesa. Respecto a lo que es una ley de la naturaleza afirma que:

debemos, por tanto, considerar una ley de la naturaleza o bien, primero, como una proposición general que anuncia, en términos abstractos, todo un grupo completo de hechos relacionados en su comportamiento de agentes naturales en determinadas circunstancias, o, segundo, como una proposición que anuncia que una clase completa de individuos que están de acuerdo con un carácter determinado están también de acuerdo con otro. (Herschel 1987, p. 100)

De esas dos formas básicas piensa que la segunda nos guía directamente a considerar, si no la causa última, sí la causa próxima, ${ }^{6}$ en el sentido de que siempre que observamos dos fenómenos invariablemente conectados uno con otro, concluimos que de hecho están relacionados uno con otro, ya sea en una relación causa-efecto, o como efecto común de una misma causa. De esa forma, las leyes son para Herschel enunciados que expresan relaciones constantes entre causa y efecto; esa relación es para Herschel real. Es ilustrativo que el ejemplo utilizado por Herschel para mostrar este doble significado de lo que considera una ley de la naturaleza sea el fenómeno de la doble refracción, en donde hay justamente relaciones constantes. Este tipo de leyes suponen una ontología de sucesos, no de procesos; es decir, cada vez que un suceso ocurre, se espera que otro suceso específico se presente. Los procesos son construidos mediante una serie de sucesos y puede haber casos en que se relacionen de diversas maneras. Como veremos, Whewell construirá su idea de causalidad histórica sobre la base de esa diferencia. El ejemplo que proporciona Herschel para mostrar la relación causal como

6 Estas últimas son las causas más inmediatas al fenómeno que queremos explicar, mientras que las primeras son aquellas que encontramos en fenómenos que ya no es posible seguir analizando. 
él la entiende es el de la doble refracción, el cual es un claro caso en donde el suceso por explicar es gobernado por leyes de la naturaleza siempre de la misma forma. Esto lo expresa Herschel en los siguientes términos:

cada ley es una provisión para casos que pueden ocurrir y tiene relación con un número infinito de casos que nunca han ocurrido y que nunca ocurrirán. Ahora, es esta provisión, a priori, de contingencias, esta contemplación de posibles ocurrencias, $[\ldots]$ que nos impresiona con la noción de una ley y una causa. (Herschel 1987, p. 36)

Estas consideraciones nos llevan a otra diferencia respecto a Whewell, a saber, la idea de unidad de la ciencia de Herschel. Para este último, es posible aplicar la idea de causalidad de manera general a todas las ramas de la ciencia, incluyendo a la geología. En términos generales, tal unidad se da en términos de relaciones mutuas entre las diferentes ramas de la ciencia. Por ejemplo, el caso de la mineralogía es particularmente ilustrativo a este respecto porque en ella "no hay rama de la ciencia que presente tantos puntos de contacto con otros departamentos de la investigación física y sirva como una conexión entre tantos puntos muy distantes de la especulación filosófica" (Herschel 1987, p. 290). La unidad de la ciencia según Herschel se articula mediante relaciones de diferentes conocimientos obtenidos en áreas diversas, y en esa medida es una unidad epistemológica y metodológica. Epistemológica en parte porque la manera en que son explicados diferentes fenómenos de diversas disciplinas es la misma: "nuestro objetivo es desenmarañar, arreglar, y presentarlos en un estado separado y distinto: y para este fin estamos llamados a resolver el importante pero complicado problema, a saber, dado el efecto o conglomerado de efectos, encontrar las causas" (Herschel 1987, pp. 221-222). Metodológica porque su concepción del método de la vera causa ha de aplicarse en las diversas ciencias.

\section{Una distinción fundamental: ciencias mecánicas y ciencias palætiológicas}

Whewell evalúa la teoría geológica de Lyell a la luz de su teoría de la ciencia en su History of Inductive Sciences de 1837 y en su Philosophy of Inductive Sciences de 1840. En su History mantiene que la geología es una de las ciencias llamadas por él palætiológicas:

La geología, formando una clase palætiológica de ciencia misma que traza hacia atrás la historia de la Tierra y sus habitantes sobre bases filosóficas, está asociada con un gran número de otros tipos de investigaciones, como el lenguaje, la ley, el arte y consecuentemente con las facultades internas del hombre, sus pensamientos sus hábitos sociales su concepción del derecho, su amor por la belleza, etc. La geología está así dentro de la atmósfera de las especulaciones 
morales y mentales; puede esperarse que sus investigaciones del pasado probable compartirán una influencia común con ellas y que no se le permitirá señalar un origen por ella misma, un comienzo meramente físico de las cosas; sino que, tanto como ella se aproxima hacia esa meta, será guiada a constatar que el punto de convergencia de muchas líneas es el origen de muchas cadenas de sucesos. (1857, no. 3, p. 484)

Pero ¿qué son exactamente las ciencias palætiológicas para Whewell? ise refiere sólo a un subgrupo de ciencias con características similares a las ciencias no-palætiológicas o a un grupo autónomo en algún sentido? En una primera caracterización, Whewell sostiene que estas ciencias son "aquellas cuyo objetivo es ascender del estado presente de las cosas hasta una condición más antigua, de la cual el presente es derivado mediante causas inteligibles"7 (Whewell 1857, p. 637). En este tipo de ciencias, uno de los rasgos epistemológicos básicos es trazar causalmente la historia de ciertos fenómenos. El punto crucial, en la medida en que marca una diferencia clara respecto a Herschel, es la manera en que entiende la expresión "causas inteligibles". Para arrojar alguna luz sobre ésta podemos empezar analizando algunas ideas básicas de Whewell.

Whewell distingue dos tipos de causalidad: la mecánica ligada a las ciencias Mecánicas y la histórica concerniente a las ciencias Palætiológicas. Adelante veremos en detalle estas diferencias. Articula el término "palætiológico" partiendo del término "ætio" que viene del griego y significa una causa y de "paleo" que significa antiguo. Del término ætio sostiene que:

Pero ese término [ætio] no describe suficientemente las especulaciones sobre las cuales estamos hablando, ya que éste puede incluir ciencias que tratan de Causalidad Permanente, como la Mecánica, como también investigaciones concernientes a la Causalidad Progresiva. (Whewell 1837, p. 397)

En su Philosophy afirma que:

Mientras la Palæontología describe a los seres que han vivido desde épocas muy antiguas sin investigar sus causas, y la Etiología trata a las causas sin distinguir la causalidad histórica de la mecánica, la Palætiología es una combinación de las dos ciencias. Explora mediante los medios de la segunda, los fenómenos presentados por la primera. (Whewell 1857, p. 638)

Debemos advertir que Whewell entiende "causalidad progresiva" y "causalidad histórica" como sinónimos. Para entender la diferencia entre causalidad

7 Qué exactamente quiere decir con "to ascend" es algo que ha sido parcialmente elucidado (Quincy 1988, p. 293). 
mecánica e histórica es importante observar que una de las metas de las ciencias palætiológicas es hacer inteligible el cambio que se presenta en los fenómenos que estas ciencias estudian, el cual es un cambio causal. La causalidad progresiva es justamente progresiva en el sentido de hacer inteligibles las causas que han llevado a un proceso histórico al estado actual. ${ }^{8}$ En este caso se quiere dar cuenta de la posible serie de sucesos mediante los cuales el estado presente llegó a ser lo que es. Para Whewell la causalidad progresiva se determina con base en "sucesos reales del pasado". Estos sucesos reales del pasado con que contamos son los datos mediante los cuales se realiza la reconstrucción histórica que permita conocer parcialmente por qué un estado de cosas llegó a su actual constitución. En geología, por ejemplo, el registro fósil, el templo de Serapis, las divisiones estratigráficas, etc., representan sucesos reales del pasado a partir de los cuales construir explicaciones. Más adelante me detendré en este punto ya que representa una de las principales críticas de Whewell a la geología.

Encontramos una explícita y clara diferencia entre la causalidad mecánica y la progresiva en un parágrafo de su Philosophy:

Hemos ya considerado las ciencias que dependen principalmente de la Idea de Causa, a saber, las Ciencias Mecánicas. Pero es obvio que la idea de Causa en las investigaciones ahora en consideración [palætiológicas] deben emplearla de una manera muy diferente que aquellas en que la aplicamos originalmente. La Fuerza es la causa del movimiento porque la fuerza en todo tiempo y bajo cualquier circunstancia, si no es interrumpida, produce movimiento. Pero la causa de la presente condición y elevación de los Alpes, cualquiera que haya sido, se manifestó en una serie de sucesos de los cuales cada uno acaece a su vez y ocupó su propio lugar en una serie de tiempo. La primera es una causa mecánica; la segunda, histórica. (Whewell 1857, p. 654)

La causalidad mecánica es determinista y ahistórica, en el sentido de que en todo tiempo y bajo cualquier circunstancia, que no la impida, produce el efecto esperado. Éste es justamente el sentido de causalidad sostenido por Herschel. En contraposición, Whewell entiende la causalidad progresiva como una cadena causal que se extiende desde casi el principio de ias cosas hasta el momento presente, en donde el pasado ha sido una serie de sucesos conectados por esta causalidad y el presente es el último término de la serie. Cada ocurrencia que haya tenido lugar en cualquier proceso histórico -como la historia del sistema solar, de la Tierra o de sus criaturas- ha sido al mismo tiempo causa y efecto; el efecto del suceso precedente y la causa

8 Un "proceso histórico" supone un tiempo humanamente grande. Por eiemplo, algunas reacciones químicas, si bien necesitan tiempo para llevarse a cabo, no por ello se tendrian que considerar como proceso histórico en el sentido aquí sostenido. 
del siguiente, así la idea de cadena causal no es sólo una metáfora, sino la forma sustancial en que opera este tipo de causalidad.

Justo aquí nos encontramos con aquello que Whewell consideró como un problema crucial de las ciencias palætiológicas, a saber cómo determinar la manera en que cada eslabón de la cadena causal es derivado del precedente y si es posible calcular hacia atrás la sucesión hasta el origen de la serie. Una forma de plantear este problema es en términos de cómo determinar el efecto de una causa histórica o progresiva, dificultad que supone a su vez el problema de cómo individualizar una causa histórica. Whewell plantea este problema general en geología de la siguiente manera:

Por ejemplo, entre las corrientes de lava que trazamos como parte de las playas de Italia y Sicilia, ¿cuáles hemos de seleccionar como correspondientes al orden existente de las cosas? Yendo hacia atrás en el tiempo, ¿dónde hemos de trazar la línea? y ¿por qué en tal punto particular? Estas cuestiones son importantes puesto que nuestra estimación de la eficacia de las causas conocidas variará con el grado de los efectos que les adscribimos. (Whewell 1857, p. 658; las cursivas son del autor)

El problema epistemológico de la individualización surge de la imposibilidad de establecer la eficacia de las causas conocidas. En otras palabras, conocer algunas causas del pasado supone individualizarlas y así saber cuáles son los efectos que legítimamente les podemos atribuir. El punto de Whewell es precisamente que en estas ciencias no hay bases seguras para asignar efectos cuando razonamos de los efectos a las causas. Determinar los efectos de las causas geológicas está en el centro de las controversias entre diferentes teorías geológicas: "las controversias de las cuales varias teorías de los geólogos han surgido, procediendo en varias formas sobre los efectos de las causas reales del cambio, conducen a los hombres a observar [...] la actual operación de tales causas" (Whewell 1837, p. 452). Aquí Whewell parece inclinarse hacia el actualismo, pero al mismo tiempo sugiere implícitamente que el actualismo es insuficiente para considerar confiable una teoría geológica, en la medida en que el problema de determinar exactamente el efecto de la causa geológica es un problema más fundamental y que recurrir a causas actualmente existentes en ocasiones no lo resuelve. Es un problema que produce controversias entre teorías geológicas rivales.

Otro problema directamente relacionado con éste se refiere al hecho de que cada paso sucesivo en el desarrollo de un determinado proceso histórico que se quiere explicar, se vuelve cada vez más complejo. Este segundo problema importante de las ciencias palætiológicas lo expresa Whewell de la siguiente forma: 
En todas ellas [ciencias palætiológicas] tenemos el mismo tipo de manifestaciones de un número sucesivo de cambios, cada uno emerge de un estado precedente. Y, en cada paso, todos los fenómenos se hacen más y más complicados, mediante el envolvimiento de los resultados de todos los estados precedentes, modificados mediante agentes supervinientes. (Whewell 1837, p. 399)

Un ejemplo claro de esta creciente complejidad es la historia de los idiomas. Las lenguas son un conglomerado de otras lenguas, muertas o aún vivas, que varía con el paso del tiempo. No son entidades fijas como las involucradas en la causalidad mecánica en donde se sostiene que siempre que haya ciertas condiciones iniciales se dará un resultado específico. El lenguaje, las costumbres sociales, las instituciones políticas, junto con las formaciones geológicas, etc., son según Whewell, los objetos de estudio de las ciencias palætiológicas y experimentan con el paso del tiempo la superposición de formaciones, lo que los hace cada vez más complejos. Los sucesivos cambios que experimenta un proceso en las ciencias palætiológicas están articulados por sucesos —eslabones de la cadena causal- que en cada etapa sufren lo que podemos llamar ganancias y pérdidas. Estos procesos históricos no son conformados por el mismo fenómeno cambiando a lo largo del tiempo, sino que en un sentido importante Whewell está afirmando que a lo largo del tiempo estos fenómenos históricos van incorporando una nueva identidad en cada etapa del proceso histórico. Da como ejemplo el idioma inglés el cual ha ido cambiando gradualmente sus características como lengua. En la geología se presenta el mismo patrón de desarrollo histórico en donde se han sufrido constantemente una serie de cambios conectados.

Este problema de la complejidad está estrechamente ligado al de la individualización, ya que la creciente complejidad no permite establecer criterios de individualización fijos para la descripción de fenómenos gobernados por la causalidad histórica. Una descripción del inglés es diferente ahora que hace trescientos años o incluso imposible si nos vamos suficientemente atrás en el tiempo.

Enfrentadas con los problemas que plantea la causalidad progresiva o histórica, desde el punto de vista de Whewell, las ciencias palætiológicas son altamente hipotéticas, en el sentido de que la reconstrucción que se hace del pasado sólo puede ser conjetural. A pesar de que estas ciencias traten con los sucesos reales del pasado, el problema es que la gran mayoría de ellos se han perdido y no contamos con el suficiente número para elaborar teorías confiables. ${ }^{9}$ En el caso de la geología, ésta forma un tipo de progresión causal que podemos representarnos como "actuando en sucesión en una historia hipotética de la Tierra y sus habitantes" (Whewell 1857, p. 656;

9 Como veremos adelante, esto es el núcleo de la crítica de Whewell a la teoría de Lyell. 
las cursivas son del autor). El hecho de que sea una historia hipotética no representa en realidad una dificultad metodológica insalvable para Whewell quien acepta que la ciencia trabaje con hipótesis, siempre y cuando ellas cumplan con rigurosos criterios metodológicos. ${ }^{10}$

Para él, el desarrollo de las ciencias palætiológicas está constituido en tres etapas que, si bien son sucesivas, se interrelacionan. En el caso de la geología las etapas son: geología descriptiva o fenoménica, geología dinámica o causal y, finalmente la etapa de madurez teórica, la geología física. Sostiene, en referencia a los geólogos, que "antes de que ellos puedan obtener alguna Teoría Geológica sólida, deben cultivar cuidadosamente la Geología Ætiológica [etapa descriptiva]" (Whewell 1857, p. 653). Esta etapa tiene como eje metodológico la clasificación, en la medida en que "ninguna descripción de una gran y variada masa de fenómenos puede ser útil o inteligible sin clasificación" (Whewell 1857, p. 645). En la clasificación, el descubridor debe "detectar los principios verdaderos de las clases naturales y seleccionar las marcas mediante las que puedan ser reconocidas" (ibid.). En esta primera etapa, la clasificación es uno de los problemas centrales.

La segunda etapa, la de la geología dinámica, es en realidad un puente entre la etapa descriptiva y la teórica. En ésta se determinan las leyes empíricas generales que obedecen los fenómenos que fueron clasificados en la primera etapa. Esta segunda etapa es de transición y básicamente consiste en:

una ciencia que debe investigar y determinar las leyes y las consecuencias de las causas conocidas de los cambios, como las que la Geología considera (y que debe hacer esto, no de una manera ocasional, imperfecta y desconectada, sino mediante métodos sistemáticos, completos y conclusivos) debe, en síntesis, ser una Ciencia, y no un ensamble revuelto de ensayos sin entusiasmo. (Whewell 1837, p. 451)

Es crucial observar que aunque se requiera que sea una ciencia sistemática, completa y conclusiva es sólo una etapa de transición en la conformación de la geología teórica. Esta ciencia es aún una ciencia ætiológica en la media en que hace referencia a hechos particulares, por ejemplo, causas acuosas del cambio, causas ígneas, elevación de los continentes, etc. Para Whewell la teoría de Lyell está en esta etapa, sostiene que "el trabajo del Sr. Lyell [Principios de Geología] ha contribuido eminentemente a colocar a la Geología Dinámica en su propia posición sobresaliente" (ibid.). "Posición

10 Como uno de los más importantes criterios de elección de hipótesis, Whewell sostiene que las teorías verdaderas no sólo han de dar cuenta de fenómenos, sino predecirlos. Las ciencias paætiológicas no satisfacen esta prueba fundamental. 
prominente" en el sentido que Lyell apela a causas conocidas para construir una ciencia geológica causal que es fundamental en la constitución de una geología teórica o física. Adelante veremos por qué para Whewell la ciencia geológica de Lyell no puede acceder a un estatus epistemológico de geología teórica.

La tercera etapa es aquella en la cual las ciencias palætiológicas alcanzan su madurez epistemológica, i.e., son ciencias que se constituyen con teorías verdaderas. De este tercer nivel Whewell mantiene en su Philosophy que:

Podemos dar cuenta de hechos conocidos mediante causas inteligibles, podemos inferir hechos latentes de efectos manifiestos, para obtener una idea distinta en la historia entera de los sucesos hasta el tiempo presente y para ver el resultado final de la historia en la condición presente de las cosas. El término Teoría, cuando es empleado rigurosamente en las ciencias que aquí consideramos, conlleva cercanamente el sentido que he adoptado: implica un enfoque consistente y sistemático de los hechos actuales, combinado con una aprehensión verdadera de sus conexiones y causas. (Whewell 1857, p. 663)

Sólo en esa etapa de desarrollo podemos tener un amplio punto de vista de los sucesos que realmente ocurrieron. Cuando se llegue a un desarrollo perfecto de esta etapa, será una ciencia demostrativa que involucre casos generales, i.e., conocimiento geológico real y permanente, como todo el demás conocimiento físico. Pero Whewell sostenía que este nivel de desarrollo de la geología aún no se había alcanzado para 1840 y todavía más, en su History llega a mantener que ni siquiera es seguro que haya empezado el desarrollo de la geología física: "la historia de la Astronomía Física apenas comenzó con Newton y pocas personas se aventurarían a sostener que el Newton de la Geología haya aparecido" (Whewell 1837, p. 497). Lo que sostiene Whewell no es el hecho de que no hayan aparecido para 1840 teorías geológicas que pretendieran ser verdaderas y sólidas; al contrario, afirma que las teorías geológicas han sido abundantes y varias (principalmente se refiere a los catastrofistas, uniformistas, plutonistas y neptunistas). El punto importante aquí es que esas teorías generales, si bien pretenden ser caracterizadas como teorías verdaderas - conocimiento geológico físico, en la mayoría de ellas "no trazamos ese carácter".

Las principales críticas que tiene Whewell para sostener la ausencia de una teoría geológica física verdadera son básicamente razones epistemológicas tomadas del proceso de desarrollo de las ciencias en general. En primer lugar, las teorías geológicas que pretenden ser teorias físicas como las de los neptunistas y plutonistas sólo han sido teorías prematuras en la medida en que se apoyan en casos limitados y en circunstancias no generales. El hecho, por ejemplo, de que la división de las rocas en primitivas, secundarias y ter- 
ciarias pueda elaborarse con base en los trazos de los antiguos seres vivos que quedaron impresos en ellas, o el hecho de establecer el origen ígneo de algunas rocas, etc., si bien todo ello constituye verdades geológicas, no es suficiente para poder establecer una teoría geológica general, tal como lo pretenden ambas posiciones. Estas dos teorias generales se esforzaban, considera Whewell, en dar una completa y simple explicación de todos los hechos de la historia de la Tierra tan sólo con los limitados elementos con que contaban. Werner, el principal defensor de los neptunistas, sólo procedió a examinar un pequeño distrito de Alemania para elaborar su teoría general, mientras que Hutton, defensor de los plutonistas, analizando sólo los estratos de Escocia e Inglaterra, pensó que estaba justificado en generalizar principios de acción volcánica para la elevación de continentes (Whewell 1837, p. 503). El argumento que subyace en estas críticas de Whewell es que estas teorías aun no habían desarrollado una geología descriptiva y pretendían, no obstante, erigirse como conocimiento geológico verdadero y permanente, i.e., como la geología física.

Respecto a las teorías catastrofistas y uniformistas, Whewell más bien se inclinaba por las tesis catastrofistas. Su principal razón era el registro fósil, considerado por muchos en la época, entre ellos Whewell, como evidencia de la existencia de grandes catástrofes, debido a que en cada nivel de tal registro había restos fosilizados de especies diferentes, lo cual era interpretado como extinciones y creaciones de nuevas especies. La pregunta por la desaparición de tales especies era contestada justamente por medio de grandes catástrofes, mientras que la cuestión de la creación de nuevas era respondida mediante parte del argumento del diseño. Este argumento se basa en la idea de que el Creador origina o diseña nuevas especies de una manera tal que éstas estén perfectamente bien adaptadas. Whewell pensaba, junto con algunos de sus contemporáneos, que la perfecta adaptación de la mayoría de las especies a sus hábitats, una cuestión crucial de la biología de la época, era un asunto de Dios. No obstante, para Whewell las teorías catastrofistas no tienen un estatus de teoría geológica física, principalmente porque él considera el catastrofismo como una tesis general sobre un aspecto geológico que no provee de una explicación palætiológica.

\section{Diferencias ontológicas de un asunto metodológico: Whewell y Herschel respecto a Lyell}

Las razones que expone Whewell en contra de los uniformistas, y particularmente de Lyell, han de analizarse con detenimiento ya que aquí están claramente expuestas algunas divergencias metodológicas y epistemológicas respecto a Herschel. En prirner lugar, Whewell sostiene, Lyell intenta 
plantear el uniformismo "por referencia a Causas ahora en Operación" (Whewell 1837, p. 511). Whewell subraya el subtítulo que Lyell utilizó en sus Principles. ¿Cuál es la razón de ese énfasis en las causas ahora en operación? La respuesta puede ser doble. $O$ bien Whewell enfatizaba positivamente el hecho de que Lyell apelara a causas conocidas, contrariamente a lo que hacían las teorías rivales, o bien enfatizaba negativamente el hecho de que Lyell intentara elaborar explicaciones de fenómenos geológicos acudiendo sólo a causas ahora en operación. Hay dos fuertes razones para rechazar la primera opción y aceptar la segunda. En primer lugar, Whewell era un partidario más o menos convencido de las catástrofes por los motivos arriba señalados y, en segundo lugar, en Whewell no hay una identificación, como en Herschel, entre catastrofistas con método hipotético y uniformistas con método por vera causa. Ambas posiciones, catastrofistas y uniformistas, son global y epistemológicamente insatisfactorias para Whewell. Veamos esto con más detalle.

En primer lugar refiriéndose al uniformismo de Lyell sostiene que "les debemos garantizar de una vez, a los defensores de ese uniformismo geológico, que no estamos suponiendo arbitrariamente la existencia de catástrofes" (Whewell 1837, p. 513; las cursivas son mías). Según Whewell, no hay razones para descartar a priori el catastrofismo como una hipótesis sin fundamento empírico, estrategia que según él siguió Lyell para descartar el catastrofismo (Whewell 1837, pp. 511-512). Las grandes catástrofes representan para Whewell, si bien no causas directamente observadas, sí inferencias fuertemente apoyadas por diferente evidencia geológica. Con ello está apelando a un principio arriba analizado: en las ciencias palætiológicas es mediante el conocimiento de los efectos que llegamos a determinar las causas. El principio seguido por Lyell, sostiene Whewell, es evitar todo prejuicio sobre las fuerzas que se desvían en tipo y grado de aquellas que actúan en el presente. Pero:

cuando el Sr. Lyell va más lejos y lo considera [dicho principio] un mérito en el curso de la especulación geológica y rechaza cualquier diferencia entre la intensidad de las causas del pasado y de las actuales, concebimos que él yerra no menos que lo que censura. "Un esfuerzo sincero y paciente para reconciliar los prístinos indicios del cambio" (Lyell 1830, vol. III, p. 328) con cualquier clase restringida de causas - un hábito que disfruta- no es, sugerimos, el carácter en el cual la ciencia debe ser perseguida. (Whewell 1837, p. 513; las cursivas son del autor)

Whewell considera que es un camino errado para desarrollar la ciencia, particularmente si estamos tratando con ciencias palætiológicas, en la medida en que son los efectos los que nos indican la naturaleza y la in- 
tensidad de las causas. Con ese proceder de Lyell estamos violando, según Whewell, principios metodológicos básicos de la geología dinámica. Aquí Whewell añade una consideración más: la acumulación en el tiempo de la eficacia de las causas puede sin lugar a dudas hacer mucho en favor del teórico en geología, pero la fuerza, de la cual no podemos medir sus límites ni llegar a comprender su naturaleza, es también un poder que no podemos menospreciar (Whewell 1837, p. 513).

Whewell enfatiza el error metodológico cometido por Lyell en los siguientes términos:

En verdad, conocemos causas sólo por sus efectos y con el fin de aprender la naturaleza de las causas que modifican la Tierra, debemos estudiarlas a través de todas las épocas de su acción. No [debemos] seleccionar arbitrariamente el periodo en que vivimos como el estándar de todas las otras épocas. Las fuerzas que han producido los Alpes y los Andes las conocemos mediante la experiencia, no menos que las fuerzas que han levantado el Etna hasta su altura presente; aprendemos sus cantidades en ambos casos por sus resultados. Entonces, ipor qué hacemos un mérito del uso del caso último como una medida del primero? O, ¿cómo podemos conocer la escala verdadera de tal fuerza, excepto por comprehensión según creemos, de todos los hechos que podamos acumular? (Whewell 1837, p. 514)

Es interesante ver la forma en que Whewell acusa a Lyell de lo mismo que este último critica del catastrofismo y por lo cual lo rechaza. Lyell está eligiendo un periodo geológico -el presente-, a los ojos de Whewell, arbitrariamente para explicar la totalidad de los fenómenos del pasado. "Arbitrariamente" puesto que es accidental que Lyell viviera en cierto periodo geológico, si hubiera vivido en un periodo de grandes catástrofes geológicas, otras hubieran sido las causas propuestas por él. Si Lyell critica del catastrofismo la elección arbitraria —en el sentido de mera hipótesisde las grandes catástrofes como causas reales, su misma elección de las causas ahora operando es igualmente arbitraria. Otra de las críticas de Whewell surgida de su idea de lo que ha de ser el desarrollo de las ciencias palætiológicas, es que Lyell construye su teoría geológica sin antes tener una sólida geología dinámica. Arriba vimos que más bien la evaluación de Whewell del trabajo geológico de Lyell es colocarlo como un buen avance justamente de la geología dinámica. Es crucial hacer notar que Whewell no critica la posibilidad de que su teoría fuera verdadera, sino que de hecho no podemos saber, a la luz de la evidencia con que Lyell cuenta, si la escala de la fuerza a la que apela es verdadera. No se tiene la evidencia suficiente -tarea que le compete a la geología dinámica - para conocer la verdadera dimensión de las fuerzas geológicas. No sabemos cuál sea el límite de la 
fuerza de lạs catástrofes que debemos permitir en una teoría como la de Lyell.

Pero la teoría de Lyell tiene aún otros problemas serios según Whewell. Recurrir a causas conocidas no es ninguna garantía de que se alcance un conocimiento sólido en las ciencias palætiológicas. Whewell plantea que, siguiendo el razonamiento de Lyell, podemos asumir similitudes entre los principios que originaron los procesos de formación de los lenguajes, gobiernos o sociedades, en etapas tempranas, y los procesos ahora en operación. Pero para Whewell es claro que no podemos hacer eso, se pregunta:

¿dónde encontramos ahora un lenguaje en proceso de formación, desarrollándose en inflexiones, terminaciones, cambios de vocales mediante relaciones gramaticales, tal como se caracterizan los más antiguos lenguajes conocidos? ¿Dónde vemos una nación, mediante sus facultades naturales, escritura inventada o las artes de la vida, como las encontramos en muchas de las antiguas naciones civilizadas? (Whewell 1837, p. 516)

Los procesos de formación de fenómenos históricos fueron diferentes de los que vemos ahora. En un sentido importante, el intento de Lyell de extender los estándares metodológicos de vera causa de la teoría celeste newtoniana a una teoría geológica, está destinado al fracaso. "La astronomía, como la ciencia de los movimientos cíclicos, no tiene nada en común con la Geología" (Whewell 1837, p. 516), en la medida en que la primera no es una ciencia palætiológica.

Whewell estaba sugiriendo que la noción de causa de las ciencias mecánicas no podía utilizarse en las explicaciones de teorfas palætiológicas. Whewell elaboró otro argumento general en contra de utilizar la analogía entre astronomía y ciencias palætiológicas, en términos de que ambas apelan a causas conocidas, tal como Lyell lo intenta. Esta vez se trata de un error en la apreciación del desarrollo histórico de la astronomía. Whewell se pregunta si el mérito de los fundadores de la astronomía está realmente en asumir que las revoluciones celestes resultaban de la elección de causas conocidas. Menciona que cuando Newton por primera vez intentó explicar los movimientos de la Luna por la fuerza de la gravedad, falló porque las medidas que utilizó eran erróneas. ${ }^{11}$ Ante esta falla Whewell se pregunta

11 Newton dice que para 1666, año que él pasa en Woolsthorpe por causa de la peste, ya había llegado a la conclusión de que la gravedad se extiende hasta la órbita de la Luna, de lo cual deduce que la fuerza que mantiene a la Luna en su órbita varía inversamente al cuadrado de la distancia. Sin embargo, tarda 21 años antes de publicar estos resultados. En una conversación con Pemberton hacia finales de su vida, declaró que, no contando con libros en Woolsthorpe, estimó un grado como 60 millas en lugar de la medida más adecuada de 69.5 millas. 
si habría sido correcto por parte de Newton insistir en que la diferencia encontrada debía ser pasada por alto, porque de otra manera deberíamos estar obligados a recurrir a causas diferentes de aquellas usualmente registradas, o bien, se pregunta Whewell, habría alguna alabanza hacia aquellos que asumieron las fuerzas celestes en términos de la gravedad, más que a aquellos que las identificaron con cualquier otra fuerza conocida - como el magnetismo-, hasta que el cálculo correcto de las leyes y la suma de esas fuerzas determinó claramente tal identificación (Whewell 1837, p. 515). Whewell sostiene que no en todo momento es posible apelar a causas conocidas, que incluso algunas de las que ahora son tenidas como tales, tropezaron en su descubrimiento con dificultades no resueltas sólo en términos de causas conocidas.

\section{Conclusión}

Para Herschel la teoría de Lyell, aunque incompleta, representaba una teoría legítima, elaborada bajo los estándares de la metodología de causas verdaderas. Contrariamente, Whewell la consideraba como una teoría con diversos problemas importantes, tales como la falta de evidencia para construir una teoría geológica física, la individuación de las causas, la determinación de sus efectos, etc. Estas diferencias descansan en algunos puntos de controversia entre Herschel y Whewell respecto a cómo entendían el desarrollo de la metodología de las causas verdaderas. En primer lugar, la noción y el papel de las "hipótesis" en geología es fuente de diferencias. Mientras que para Herschel y Lyell, apelar a grandes catástrofes, como lo hacían los catastrofistas, era recurrir a hipótesis y, por tanto, se incurría en conocimiento no seguro, para Whewell no había razones sólidas para descartar grandes catástrofes como recurso explicativo, en la medida en que la evidencia geológica apuntaba en su favor. Bajo qué criterios sostener que las grandes catástrofes tienen carácter hipotético, era el punto en discusión, el cual no era posible dirimir recurriendo a la evidencia disponible, sino que se analizaba respecto a lo que cada uno aceptaba como concepción legítima de ciencia. La idea general de ciencia y su concepción de desarrollo histórico determinó en gran medida la evaluación de la teoría de Lyell.

Otro punto de diferencia entre Herschel y Whewell fue la noción de causalidad que interviene en la geología; mientras que para Herschel dicha noción no es diferente en tipo de la noción aplicada en óptica o en mecánica, para Whewell la causalidad histórica es diferente de la que exhiben las ciencias mecánicas. Asimismo el tipo de ciencia que era la geología también constituyó una fuente de diferencias respecto a qué clase de teoría desarrollar. Igualmente sus diferencias descansan en la manera de entender el desarrollo histórico de las disciplinas que involucran de alguna forma 
aspectos históricos, y en problemas tales como el papel de las leyes y las causas, la clasificación e individualización de las causas históricas. Estas diversas dificultades conformaron el complejo problema de la causalidad histórica. Es preciso subrayar que éste era el problema ontológico de fondo que yacía en la controversia metodológica suscitada por la teoría de Lyell. ${ }^{12}$

Estas diferencias, entre miembros de una misma comunidad científica respecto a temas sustanciales se reflejan en diferencias respecto a qué es una explicación genuina de una nueva teoría. Este análisis ha arrojado luz en favor de la tesis de que una parte importante de las evaluaciones que de hecho hacen los científicos, respecto al poder explicativo de teorías nuevas, está determinado en gran medida por consideraciones o presupuestos de tipo filosófico, tales como la manera en que entienden la historia de la metodología, la historia de su disciplina o el papel epistemológico de la causalidad.

\section{BIBLIOGRAFIA}

Blacke, R.M., C. Ducasse y F. Madden (1960), Theories of Scientific Method: The Renaissance through the Nineteenth Century, University Washington Press, Seattle. Buchwald, J.Z. (1989), The Rise of the Wave Theory of Light. Optical Theory and Experiment in the Early Nineteenth Century, University of Chicago Press, Chicago. Cannon, S. (1978), Science in Culture, Science History, Nueva York.

Cannon, W. (1960), "The Uniformitarian-Catastrophist Debate", Isis, no. 51, pp. 3855.

-(1976), "Charles Lyell, Radical Actualism and Theory", British Journal for the History of Science, no. 9, pp. 104-120.

Gillispie, C. (1951), Genesis and Geology, Cambridge University Press, Cambridge. Good, G. (1987), "John Herschel's Optical Researches and the Development of His Ideas on Method and Causality", Stud. Hist. Phil. Sci., no. 18, pp. 1-41.

Gould, S.J. (1965), "Is Uniformitarianism Necessary?", American Journal of Science, no. 263, pp. 223-228.

(1987), Time's Arrow. Time's Cycle, Universidad de Harvard, Harvard.

Greene, M. (1983), Geology in the Nineteenth Century, Cornell University Press, Ithaca-Nueva York.

Herschel, J. (1987), A Preliminary Discourse on the Study of Natural Philosophy, University of Chicago Press, Chicago.

12 En otro trabajo titulado "Dos concepciones metodológicas, Larry Laudan y Matthias Kaiser. Una crítica a la noción de regla metodológica", analizo con detalle las relaciones entre ontología y metodología de la discusión alrededor de la teorla de Lyell, aunque el objetivo principal de ese trabajo es elaborar una crítica a algunas ideas metodológicas de Laudan y de Kaiser (próxima publicación). 
Hodge, J. (1991), "The History of the Earth Life, and Man: Whewell and Palætiological Science", en Fisch, M. y S. Schaffer (comps.), W. Whewell. A Composite Portrait, Clarendon Press, Oxford.

Hutton, J. (1788), "Theory of the Earth, or an Investigation of the Laws Observable in the Composition, Dissolution, and Restoration of Land upon the Globe", Transaction of fhe Royal Society of Edinburgh, vol. I, pp. 209-304.

Laudan, L. (1981), Science and Hypothesis: Historical Essays on Scientific Methodology, Reidel, Dordrecht.

Laudan, R. (1987), From Mineralogy to Geology. The Foundations of a Science 16501830, University of Chicago Press, Chicago.

- (1991), "What's so Special about the Past?", en Nitecki, M. y D. Nitecki (comps)., History and Evolution, State University of New York Press, Nueva York.

Lyell, Charles (1830-1833), Principles of Geology, 3 vols., J. Murray, Londres.

Quincy, C. (1988), "Robert Chambers and William Whewell: A Nineteenth-Century Debate over the Origin of Language", Journal of the Hisfory of Ideas, no. 49, pp. 287-300.

Rudwick, M. (1972), The Meaning of Fossils, Macdonald, Londres.

Ruse, M. (1976), "Charles Lyell and the Philosophers of Science", British Journal for the History of Science, no. 9, pp. 121-131.

- (1977), "William Whewell and the Argument from Design", The Monist, no. 69, pp. 244-268.

- 1979), The Darwinian Revolution. Science Red in Tooth and Claw, University of Chicago Press, Chicago.

Whewell, W. (1837), Hisfory of the Inductive Sciences, 3 vols., Parker, Londres.

- (1857), The Philosophy of Inductive Sciences. Founded upon Their History, Parker, Londres. 